\title{
Cloning of the Bacillus thuringiensis serovar sotto chitinase (Schi) gene and characterization of its protein
}

\author{
Wan-Fang Zhong ${ }^{1,2}$, Ji-Chao Fang ${ }^{1}$, Ping-Zhong Cai ${ }^{2}$, Wen-Zhao $\mathrm{Yan}^{2},{\mathrm{Jie} \mathrm{Wu}^{2} \text { and Hui-Fang Guo }}^{1}$ \\ ${ }^{1}$ Jiangsu Academy of Agricultural Sciences, Institute of Plant Protection, Nanjing, Jiangsu Province, China. \\ ${ }^{2}$ Sichuan Academy of Agricultural Sciences, Biotechnological Breeding Center, Chengdu, Sichuan Province, \\ China.
}

\begin{abstract}
Chitinase plays a positive role in the pathogenicity of Bacillus thuringiensis to insect pests. We used touchdown PCR to clone the chitinase (Schi) gene from Bacillus thuringiensis serovar sotto (Bt sotto) chromosomal DNA. Our DNA sequencing analysis revealed that the Bt sotto Schi gene consists of an open reading frame (ORF) of 2067 nucleotides with codes for the chitinase precursor. We also found that the putative promoter consensus sequences (the -35 and -10 regions) of the Bt soto Schi gene are identical to those of the chiA71 gene from Bt Pakistani, the chiA74 gene from Bt kenyae and the ichi gene from Bt israelensis. The Schi chitinase precursor is 688 amino acids long with an estimated molecular mass of $75.75 \mathrm{kDa}$ and a theoretical isoelectric point of 5.74 , and contains four domains, which are, in sequence, a signal peptide, an N-terminal catalytic domain, a fibronectin type III like domain and a C-terminal chitin-binding domain. Sequence comparison and the evolutionary relationship of the Bt sotto Schi chitinase to other chitinase and chitinase-like proteins are also discussed.
\end{abstract}

Key words: Bacillus thuringiensis serovar sotto, chitinase, touchdown PCR, gene cloning, characterization analysis.

Received: December 22, 2004; Accepted: July 5, 2005.

\section{Introduction}

Chitin, a $(1,4)-\beta$-linked homopolysaccharide made up of $\mathrm{N}$-acetylglucosamine residues, is absent from vertebrates and plants but is commonly found in the exoskeletons of insects and crustaceans as well as in fungi and some algae. Chitinase (EC 3.2.1.14) is a glycosyl hydrolase that catalyzes the hydrolytic degradation of chitin, and is found in a wide variety of organisms including bacteria, fungi, invertebrates, plants and animals (Goody, 1990), but the roles of chitinases in different organisms are diverse. Bacteria produce chitinases to digest chitin for use as a carbon and energy sources, while fungi produce this enzyme to modify the important cell wall component chitin and invertebrates require chitinases for the partial degradation of old exoskeletons. In plants, however, chitinases are part of the plants defense mechanisms against fungal pathogens.

Some chitinolytic bacteria have been shown to be potential agents for the biological control of both phytopathogenic fungi and insect pests (Chernin et al., 1997; Sampson and Gooday, 1998). One of the most impor-

Send correspondence to Wan-fang Zhong. Jiangsu Academy of Agricultural Sciences, Institute of Plant Protection, Nanjing, Jiangsu Province 210014, China. E-mail: wfzhong88@yahoo.com.cn. tant insecticidal microbes used in biological control is $\mathrm{Ba}$ cillus thuringiensis (Bt), a gram-positive, rod-shaped, spore-forming bacterium that forms insecticidal protein(s) during the stationary phase of its growth cycle. Insecticidal proteins, mainly Cry (crystal) proteins, play the leading role in controlling of insect pests. It has been reported that chitinases are widely distributed in $B t$ strains and that some of the chitinase-producing strains can enhance the insecticidal activity of Bt (Liu et al., 2002), although, as far as we know, only a few chitinase genes have yet been cloned (Thamthiankul et al., 2001; Barboza-Corona et al., 2003; Zhong et al., 2003; Lin and Guan, 2004) and furthermore, the synergistic effect of purified Bt chitinase and Cry proteins has still not been quantitatively demonstrated (Barboza-Corona et al., 2003).

It is known that $B$. thuringiensis serovar sotto (Bt sotto) has strong larvicidal activity against lepidopteran pests because of Cry proteins (Zhong et al., 2004). We cloned the crylAal3 gene from Bt sotto plasmid and expressed it in Escherichia coli, but expression of the crylAal 3 gene product was low (Zhong et al., 2004). In order to further determine the synergistic action between the Bt sotto Cry1Aa13 protein and Bt sotto Schi chitinase, it is necessary to clone and sequence the Schi chitinase. This re- 
port deals with the cloning, sequencing and partial characterization of Bt sotto Schi gene.

\section{Materials and Methods}

\section{Bacterial strains, plasmids and culture condition}

We obtained Bt sotto from Dr. Dai LY (Chinese Academy of Forestry, Beijing) and grew cultures at $30^{\circ} \mathrm{C}$ in nutrient yeast extract salts medium (NYSM; consisting of $\left(\mathrm{gL}^{-1}\right)$ nutrient broth, 8; $\mathrm{CaCl}_{2} .2 \mathrm{H}_{2} \mathrm{O}, 0.103$; $\mathrm{MnCl}_{2} .4 \mathrm{H}_{2} \mathrm{O}, 0.01 ; \mathrm{MgCl}_{2} \cdot 6 \mathrm{H}_{2} \mathrm{O}, 0.203$ ) for chromosomal DNA extraction. The plasmid T-easy vector (Promega) was used as a cloning vector and Escherichia coli strain DH5 $\alpha$ as the transformation host, this strain being grown at $37^{\circ} \mathrm{C}$ in Luria-Bertani (LB) medium (containing $\left(\mathrm{gL}^{-1}\right)$ tryptone, $10 ; \mathrm{NaCl}, 10$; yeast extract, 5) and transformants in LB supplemented with $50 \mu \mathrm{g} / \mathrm{mL}$ of ampicillin.

\section{Primer design and oligonucleotide synthesis}

Oligonucleotide primers P1 (5'-GGG CCC TTT CCT CCC ATA CCA-3') and P2 (5'-GGG CCC CGA AAG CCT TTC CTA-3') were synthesized using $\beta$-cyanoethyl phosphoramidite chemistry and an Expedite Nucleic Acid Synthesis System (workstation) in TaKaRa Dalian Corporation (Dalian, China).

\section{DNA manipulation and cloning of the Bt sotto Schi gene}

Touchdown PCR avoids the need for complicated optimization of the annealing temperature of the primers by using a $0.5-1{ }^{\circ} \mathrm{C}$ sequential decrease from a high annealing temperature in the first PCR cycle to a "touchdown" annealing temperature which is then used for several more cycles (Don et al., 1991). Touchdown PCR was carried out in a TGradient Thermocycler (Biometra) using the following program: $94{ }^{\circ} \mathrm{C}$ for $3 \mathrm{~min}, 94^{\circ} \mathrm{C}$ for $1 \mathrm{~min}, 60^{\circ} \mathrm{C}$ for $1 \mathrm{~min}$ and $72{ }^{\circ} \mathrm{C}$ for $1.5 \mathrm{~min}$, followed by 24 cycles at decreasing annealing temperatures in decrements of $0.5^{\circ} \mathrm{C}$ per cycle, then 10 cycles of $1 \mathrm{~min}$ at $94^{\circ} \mathrm{C}, 1 \mathrm{~min}$ at $52^{\circ} \mathrm{C}, 1.5 \mathrm{~min}$ at $72{ }^{\circ} \mathrm{C}$, and final extension at $72{ }^{\circ} \mathrm{C}$ for $10 \mathrm{~min}$. The reaction mixture contained $10 \mathrm{mM}$ Tris- $\mathrm{HCl}(\mathrm{pH} \mathrm{8.0)}, 50 \mathrm{mM} \mathrm{KCl}$, $1.5 \mathrm{mM} \mathrm{MgCl}_{2}, 200 \mu \mathrm{M}$ dNTPs, $0.2 \mu \mathrm{M}$ of each primer, $1 \mathrm{U}$ Taq plus DNA polymerase (TaKaRa) and $30 \mathrm{ng}$ of Bt sotto total genomic DNA, extracted according to Zhong et al. (2003), as template. Small-scale plasmid isolation, transformation, restriction enzyme digestion and agarose gel electrophoresis were performed by standard methods, i.e. $1 \%(\mathrm{w} / \mathrm{v})$ agarose gel electrophoreses and purification using the Wizard SV Gel and PCR Clean-Up System (Promega), the resulting PCR product being ligated to the T-easy vector which was used to transform Escherichia coli DH5 $\alpha$ competent cells with the Schi gene. The positive recombinant clone was screened and used for nucleotide sequencing.

\section{Nucleotide sequence analysis}

The Bt sotto Schi gene nucleotide sequence was determined at least once in each strand by the dideoxy chain termination method using an ABI 377 sequencer. DNA and deduced amino acid sequences were compared with those of other chitinase genes from GenBank, using both the BLAST (http://ncbi.nlm.nih.gov/BLAST/) and CLUSTAL $\mathrm{W}$ multiple sequence alignment tool.

\section{Results}

\section{Cloning and nucleotide sequencing of the Bt sotto Schi chitinase gene}

We successfully cloned the Bt sotto Schi chitinase gene using touchdown PCR with primers $\mathrm{P} 1$ and $\mathrm{P} 2$. The $B t$ sotto Schi gene structural and flanking region sequence was deposited in the GenBank database under accession number AY129671. The manuscript has Schi gene region contains an open reading frame (ORF) with a base composition of $33.9 \%$ adenine and $28.4 \%$ thymine $(\mathrm{A}+\mathrm{T}=62.3 \%)$ and $19.7 \%$ guanine $18.0 \%$ cytosine $(\mathrm{G}+\mathrm{C}=37.7 \%)$ consisting of 2067 nucleotides encoding 688 amino acid residues with a deduced molecular weight of 75.75 Da and a theoretical isoelectric point of 5.74. The codon usage is shown in Table 1 , from which it can be seen that because of the relatively high $\mathrm{A}+\mathrm{T}$ content there is a preference for $\mathrm{A}$ and $\mathrm{T}$ at the third base position in the codons, the $\mathrm{A}: \mathrm{G}$ ratio being $3.67: 1$ and the T:C ratio 3.08:1.

The putative promoter consensus region, spanning nucleotide positions 59 to 64 (TTGAGA, -35) and 79 to 84 (TTAATA, -10) of the Schi gene are identical to those of the chiA71 gene from $B$. thuringiensis serovar Pakistani (Thamthiankul et al., 2001), the chiA74 gene from $B$. thuringiensis serovar kenyae (Barboza-Corona et al., 2003), and the ichi gene from B. thuringiensis serovar israelensis (Zhong et al., 2003). The putative promoters also show obvious homology to the -35 (TTGACA) and -10 (TATAAT) consensus sequence for an $E$. coli promoter and Bacillus subtilis $\sigma^{\mathrm{A}}$. Two stem-loop structures (one at 25 to $41 \mathrm{bp}$ and the other at 69 to $94 \mathrm{bp}$ from the TAG stop codon) of the putative transcriptional terminator were found at the 3 ' end, these structures which causing the polymerase to pause and subsequently cease transcription, similar results having been reported by us for the Bt ichi sequences (Zhong et al., 2003).

\section{Amino acid sequence analysis of the Schi}

The predicted $46 \mathrm{~N}$-terminal amino acids of the Schi chitinase precursor exhibited a typical feature of the signal peptide characteristic of Gram-positive bacteria. There was a positively charged hydrophilic N-terminal segment ending in lysine with a net charge of +3 , followed by a hydrophobic amino acid sequence. Cleavage of the signal sequence of the Schi chitinase precursor occurred at between A-46 and D-47. Interesting, the signal peptide may 
Table 1 - Amino acid codons for the Bacillus thuringiensis serovar sotto (Bt sotto) Schi gene.

\begin{tabular}{|c|c|c|c|c|c|}
\hline Amino acid* & Codon & $\mathrm{Nr}$ & Amino acid* & Codon & $\mathrm{Nr}$ \\
\hline \multirow[t]{4}{*}{ Alanine (A) } & GCG & 3 & Glutamine (Q) & CAG & 0 \\
\hline & GCA & 15 & & CAA & 17 \\
\hline & GCT & 19 & Glycine $(G)$ & GGG & 5 \\
\hline & GCC & 7 & & GGA & 19 \\
\hline \multirow[t]{6}{*}{ Arginine (R) } & AGG & 1 & & GGT & 25 \\
\hline & AGA & 1 & & GGC & 14 \\
\hline & CGG & 0 & Histidine $(\mathrm{H})$ & CAT & 5 \\
\hline & CGA & 2 & & CAC & 4 \\
\hline & CGT & 6 & Isoleucine (I) & ATA & 5 \\
\hline & CGC & 3 & & ATT & 26 \\
\hline \multirow[t]{2}{*}{ Asparagine (N) } & AAT & 46 & & ATC & 1 \\
\hline & $\mathrm{AAC}$ & 11 & Leucine (L) & TTG & 3 \\
\hline \multirow[t]{2}{*}{ Aspartic acid (D) } & GAT & 34 & & TTA & 19 \\
\hline & GAC & 9 & & CTG & 1 \\
\hline \multirow[t]{2}{*}{ Cysteine (C) } & TGT & 4 & & CTA & 11 \\
\hline & TGC & 4 & & CTT & 12 \\
\hline \multirow[t]{2}{*}{ Glutamic acid (E) } & GAG & 3 & & CTC & 6 \\
\hline & GAA & 27 & Lysine (K) & AAA & 47 \\
\hline
\end{tabular}

\begin{tabular}{lcrllr}
\hline Amino acid* & Codon & Nr & Amino acid* & Codon & Nr \\
\hline Lysine (K) & AAG & 5 & Threonine (T) & ACG & 10 \\
Methionine (M) & ATG & 7 & & ACA & 27 \\
Phenylalanine (F) & TTT & 17 & & ACT & 19 \\
& TTC & 7 & & ACC & 6 \\
Proline (P) & CCG & 1 & Tryptophan (W) & TGG & 20 \\
& CCA & 13 & Tyrosine (Y) & TAT & 21 \\
& CCT & 22 & & TAC & 12 \\
& CCC & 0 & Valine (V) & GTG & 3 \\
Serine (S) & AGT & 13 & & GTA & 14 \\
& AGC & 7 & & GTT & 15 \\
& TCG & 4 & & GTC & 4 \\
& TCA & 9 & End (.) & TGA & 0 \\
& TCT & 15 & & TAG & 1 \\
& TCC & 2 & & TAA & 0 \\
\hline
\end{tabular}

*Amino acid or punctuation one-letter code in parenthesis.

Nr: Number of residues.

also be recognized by gram-negative bacterial and eukaryotic organisms according to the SignalP server (http://www.cbs.dtu.dk/services/SignalP/). We estimated the mature Schi chitinase molecular weight to be $70.50 \mathrm{kDa}$ and the isoelectric point to be 5.55. Computer analysis of the deduced mature Schi chitinase amino acid sequence re-

vealed that it consists of three discrete domains, an N-terminal catalytic domain, a fibronectin type III-like domain (FLD) and a chitin-binding domain (ChBD).

The $B t$ Schi chitinase N-terminal region (residue 151 to 255) showed similarity to catalytic domains of chitinases belonging to glycosyl hydrolase family 18. In particular,

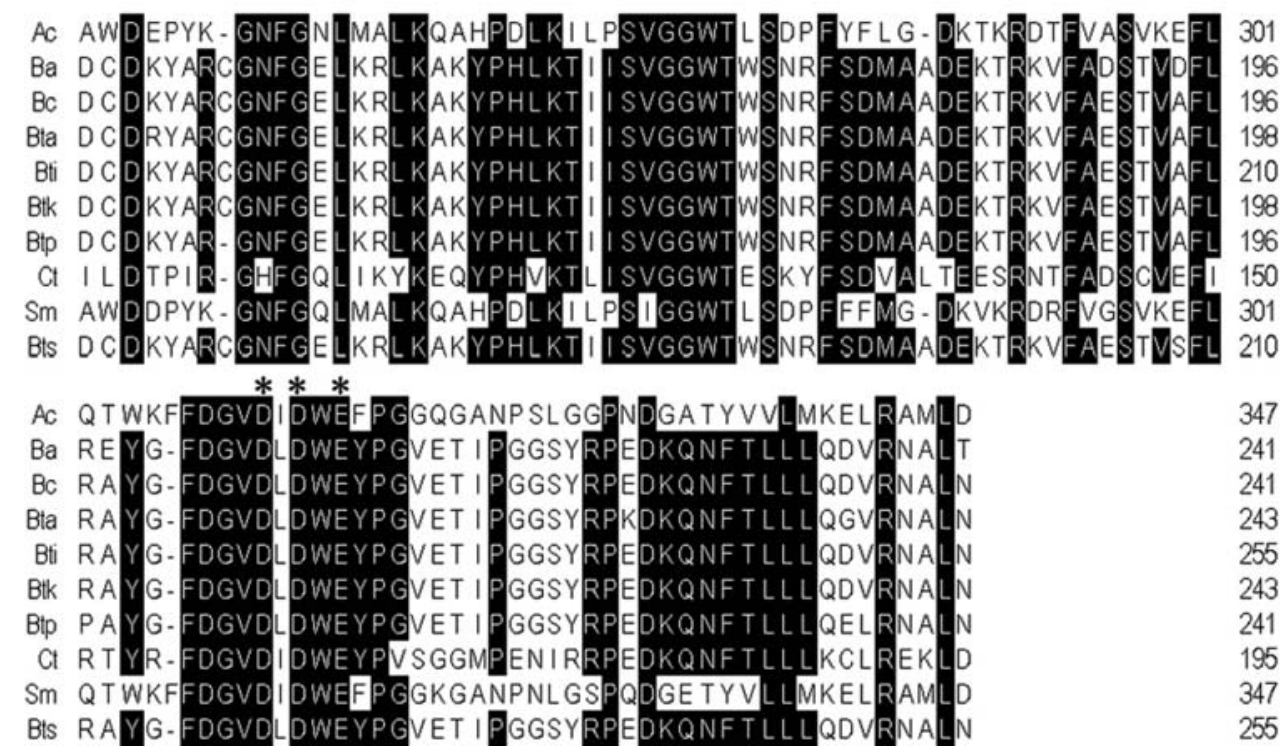

Figure 1 - Alignment of the Bacillus thuringiensis (Bt) serovar sotto (Bt sotto) Schi catalytic domain with those of other chitinases (Chi). Sequences are from Aeromonas caviae chitinase (Ac), Bacillus anthracis chitinase B (Ba), Bacillus cereus chitinase B (Bc), Bt alesti ChiA74-HD16 (Bta), Bt israelensis Ichi chitinase (Bti), Bt kenyae ChiA74 (Btk), Bt pakistani ChiA71 (Btp), Clostridium thermocellum (Ct) and Serratia marcescens ChiA (Sm). Putative proton donor amino acid residues are marked with an asterisk. Dashes indicated gaps inserted to improve alignment. Numbers refer to the amino acid residue at the end of the respective lines. 
the $\mathrm{N}$-terminal region showed high sequence similarity to the following chitinases (Chi); Bt pakistani ChiA71 (95.2\% similarity; Thamthiankul et al., 2001); Bt kenyae ChiA74 (99\%; Barboza-Corona et al., 2003); Bt alesti ChiA74HD16 (96.2\%; Lin and Guan, 2004); Bt israelensis Ichi chitinase (99\%; Zhong et al., 2003); Bacillus cereus chitinase B (99\%; Mabuchi and Araki, 2001); and Bacillus anthracis chitinase B (96.2\%; Read et al., 2003) (Figure 1). Further analysis of the $B t$ Schi chitinase showed that there was some degree of similarity between the catalytic domain of this chitinase and that of other bacterial chitinases, such as the chitinases from Aeromonas caviae (39.8\% similarity) and Clostridium thermocellum $(57.7 \%)$ as well as Serratia marcescens chitinase A (40.8\%) (Figure 1). In this region, the deduced amino acid sequence from residue 215 to 223 (FDGVDLDWE; q.v. Table 1 for amino acid key) was homologous to the active site motif of enzymes in glycosyl hydrolase family 18 ([FILMVY]-[DN]-G[VFILM]-[DN]-[LFIMV]-[DN]-X-E; q.v. Table 1 for amino acid key). Furthermore, amino acid residues D-219, D-221 and E223 of the Schi chitinase (corresponding to D-200, D-202 and E204 of Bacillus circulans chitinase ChiA) were well conserved and may pay an essential role in chitinase activity.

The Bt Schi chitinase middle region (residues 502 to $575)$ showed similarity to the fibronectin type III-like domain (FLD) sequences found in the R-1 and R-2 regions of Bacillus circulans chitinase A1 (44\% and 48.8\% similarity respectively; Watanabe et al., 1990) and also to the FLD1 and FLD2 regions of Bt Chi74 chitinase (13.1\% and 94\% respectively; Barboza-Corona et al., 2003) as well as to regions in the Bt ChiA71 chitinase $(86.9 \%$; Thamthiankul et al., 2001) and the Bt Ichi chitinase (95.2\%; Zhong et al., 2003). Interesting, the middle region of the Bt Schi chitinase were identity to the FLD of Bt alesti chitinase ChiA74-HD16 (Lin and Guan, 2004) (Figure 2).

The Bt Schi chitinase C-terminal region (residues 600 to 641 ) showed sequence homology to the chitin-binding domain found in the following chitinases: Chi71 (residues 454 to 495; Thamthiankul et al., 2001); Chi74 (residues 588 to 629; Barboza-Corona et al., 2003); Bt Ichi (residues 600 to 641; Zhong et al., 2003); ChiA74-HD16 (residues 588 to 629; Lin and Guan, 2004); Ac1 and Ac2 of Aeromonas caviae ChiA (residues 770 to 813 and 817 to 859 respectively; Sitrit et al., 1995); Alteromonas sp. strain O-7 Chi85 (residues 776 to 818; Tsujibo et al., 1993) and Vibrio harveyi ChiA (residues 512 to 557; Svitil and Kirchman, 1998) (Figure 3).

\section{Discussion}

According to our catalytic domain analysis, the $B t$ chitinase Schi gene we cloned and sequenced during this study seems to be the chiA gene encoding the chitinase precursor. The phenomenon of possible promoter showed homology with $B$. subtilis $\sigma^{\mathrm{A}}$ indicated that the expression of Schi take place during the vegetative (i.e. exponential) growth phase.

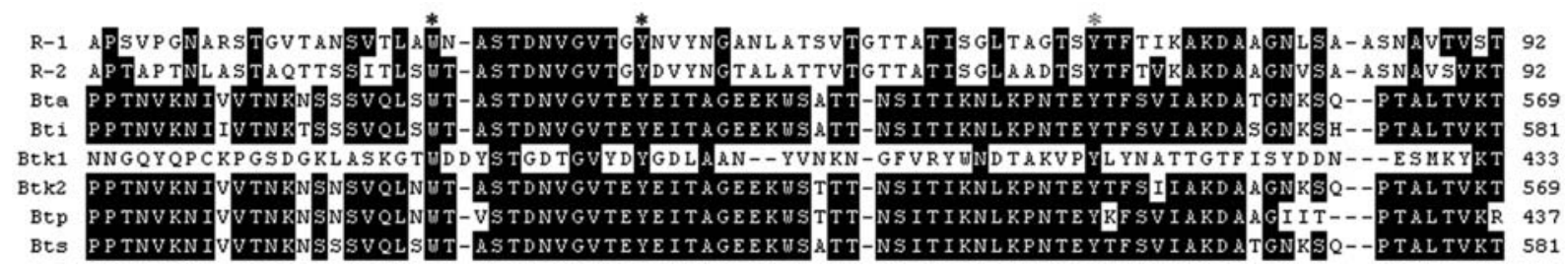

Figure 2 - Alignment of the fibrinectin type III-like domain (FLD). The sequences of the FLD domain of the Bacillus thuringiensis (Bt) serovar sotto (Bt sotto) chitinase (Chi) Schi (Bts) are aligned with those of Bacillus circulans chitinase A1 (R-1 and R-2), Bt alesti ChiA74-HD16 (Bta), Bt israelensis Ichi chitinase (Bti), Bt kenyae ChiA74 (Btk1 and Btk2) and Bt pakistani ChiA71 (Btp). Aromatic amino acid residues typical for FLD regions are indicated by an asterisk.

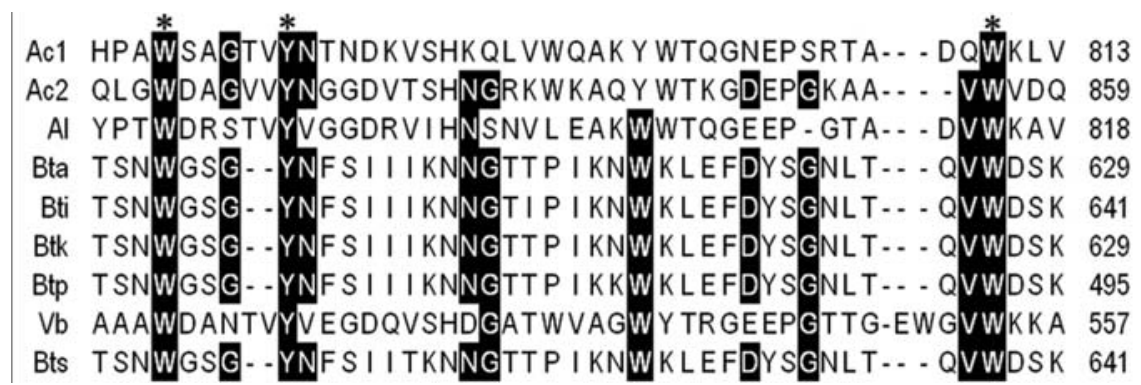

Figure 3 - Composition of the C-terminal domain of Bacillus thuringiensis (Bt) serovar sotto (Bt sotto) chitinase (Chi) Schi with chitin-binding domain (ChBD) of other chitinase. Sequences are from Aeromonas caviae ChiA (Ac1 and Ac2), Alteromonas sp. strain O-7 Chi85 (Al), Bt alesti ChiA74-HD16 (Bta), Bt israelensis Ichi (Bti), Bt kenyae ChiA74 (Btk), Bt pakistani ChiA71 (Btp) and Vibrio harveyi ChiA (Vb). Highly conserved aromatic amino acids are marked with an asterisk. 
The signal sequence of the Bt sotto Schi chitinase precursor contains an extremely long N-terminal hydrophilic segment, with the signal peptide cleavage in the Schi precursor occurring between amino acid residues A-46 and $\mathrm{D}-47$. The signal peptide is the same length as that of the $\mathrm{Bt}$ ichi chitinase but longer than that of the chiA71 and chiA74 chitinases.

The high homology (97\% identity) between the $B t$ sotto Schi chitinase and the B. cereus strain $\mathrm{CH}$ chiB chitinase (Mabuchi and Araki, 2001) supports the view that even though $B$. thuringiensis and $B$. cereus belong to different species they are closely related evolutionary.

The mature Bt sotto Schi chitinase seems to be composed of a glycosyl-hydrolase family 18 catalytic domain, a fibronectin type III-like domain and a chitin-binding domain. Chitinases are acid-base catalysts and from sequence comparison with $B$. circulans chitinase A1 (Watanabe et $a l ., 1994)$, it seems that the catalytic residues acting as proton donors are the amino acid residues D-219, D-221 and E-223. Fibronectin is a multifunctional extracellular matrix and plasma protein that plays a significant role in cell adhesion. Fibronectin type III-like domains (FLD) have been found in chitinase, cellulases, $\alpha$-amylase, and poly-3hydroxybutyrate (PHB) depolymerase. In the chitinbinding domain (ChBD) of many chitinase, the aromatic amino acids tryptophan (W) and tyrosine (Y) are highly conserved and may play a crucial role during binding to the pyranosyl rings of $\mathrm{N}$-acetylglucosamine residues in chitin (Morimoto et al., 1997). In Bt sotto Schi chitinase we found that tryptophan and tyrosine residues (e.g. W-603, Y-607, W-624 and W-638) were well conserved. It is also known that Schi chitinases show homology with the cellulosebinding domain of some cellulases and some researchers consider that chitinases and these cellulases have common substrate-binding mechanisms (Morimoto et al., 1997). The work presented in this paper has partially elucidated the role of each domain but the structure of the Bt sotto Schi chitinase is still not fully understood because of the limitations inherent in attempting to characterization of enzyme from amino acid sequences.

\section{Acknowledgments}

This work was supported by the National Basic Research Program of China (n. 2003CB114400), National Programs for Science and Technology Development of China (n. 2004BA516A02), National Natural Science Foundation of China (General Program, n. 30500341) and the Natural Science Foundation of Jiangsu Province (n. BK2002207, BK2005214). Part of this study was carried out in Biotechnological Breeding Center, Sichuan Academy of Agricultural Sciences. We would like to thank all staff members of the Institute of Biology and Nuclear Technology, Sichuan Academy of Agricultural Sciences for their kind offer of the facilities.

\section{References}

Barboza-Corona JE, Nieto-Mazzocco E, Velazquez-Robledo R, Salcedo-Hernandez R, Bautista M, Jimenez B and Ibarra JE (2003) Cloning, sequencing, and expression of the chitinase gene chiA74 from Bacillus thuringiensis. Appl Environ Microbiol 69:1023-1029.

Chernin LS, De la Fuente L, Sobolev V, Haran S, Vorgias CE, Oppenheim AB and Chet I (1997) Molecular cloning, structural analysis, and expression in Escherichia coli of a chitinase gene from Enterobacter agglomerans. Appl Environ Microbiol 63:834-839.

Don RH, Cox PT, Wainwright BJ, Baker K and Mattick JS (1991) "Touchdown" PCR to circumvent spurious priming during gene amplification. Nucl Acids Res 19:4008.

Gooday GW (1990) The ecology of chitin decomposition. Adv Microb Ecol 11:387-430.

Liu M, Cai QX, Liu HZ, Zhang BH, Yan JP and Yuan ZM (2002) Chitinolytic activities in Bacillus thuringiensis and their synergistic effects on larvicidal activity. J Appl Microbiol 93:374-379.

Lin Y and Guan X (2004) Molecular cloning and sequence analysis of the chitinase gene from Bacillus thuringiensis serovar alesti. Biotechnol Lett 26:635-639.

Mabuchi N and Araki Y (2001) Cloning and sequencing of two genes encoding chitinases A and B from Bacillus cereus $\mathrm{CH}$. Can J Microbiol 47:895-902.

Morimoto K, Karita S, Kimura T, Sakka K and Ohmiya K (1997) Cloning, sequencing, and expression of the gene encoding Clostridium paraputrificum chitinase $\mathrm{ChiB}$ and analysis of the functions of novel cadherin-like domains and a chitin-binding domain. J Bacteriol 179:7306-7314.

Read TD, Peterson SN, Tourasse N, Baillie LW, Paulsen IT, Nelson KE, Tettelin H, Fouts DE, Eisen JA, Gill SR, Holtzapple EK, Okstad OA, Helgason E, Rilstone J, Wu M, Kolonay JF, Beanan MJ, Dodson RJ, Brinkac LM, Gwinn M, DeBoy RT, Madpu R, Daugherty SC, Durkin AS, Haft DH, Nelson WC, Peterson JD, Pop M, Khouri HM, Radune D, Benton JL, Mahamoud Y, Jiang L, Hance IR, Weidman JF, Berry KJ, Plaut RD, Wolf AM, Watkins KL, Nierman WC, Hazen A, Cline R, Redmond C, Thwaite JE, White O, Salzberg SL, Thomason B, Friedlander AM, Koehler TM, Hanna PC, Kolsto AB and Fraser CM (2003) The genome sequence of Bacillus anthracis Ames and comparison to closely related bacteria. Nature 423:81-86.

Sampson MN and Gooday GW (1998) Involvement of chitinase of Bacillus thuringiensis during pathogenesis in insects. Microbiology 144:2189-2194.

Sitrit Y, Vorgias CE, Chet I and Oppenheim AB (1995) Cloning and primary structure of the chiA gene from Aeromonas caviae. J Bacteriol 177:4187-4189.

Svitil AL and Kirchman DL (1998) A chitin-binding domain in a marine bacterial chitinase and other microbial chitinases: Implications for the ecology and evolution of $1,4-\beta$ glycanases. Microbiology 144:1299-1308.

Thamthiankul S, Suan-Ngay S, Tantimavanich S and Panbangred W (2001) Chitinase from Bacillus thuringiensis serovar. pakistani. Appl Microbiol Biotechnol 56:395-401.

Tsujibo H, Orikoshi H, Tanno H, Fujimoto K, Miyamoto K, Imada C, Okami Y and Inamori Y (1993) Cloning, sequence, and expression of a chitinase gene from a marine bacterium, Alteromonas sp. strain O-7. J Bacteriol 175:176181. 
Watanabe T, Ito Y, Yamada T, Hashimoto M, Sekine S and Tanaka H (1994) The roles of the C-terminal domain and type III domains of chitinase A1 from Bacillus circulans WL-12 in chitin degradation. J Bacteriol 176:4465-4472.

Watanabe T, Suzuki K, Oyanagi W, Ohnishi K and Tanaka H (1990) Gene cloning of chitinase A1 from Bacillus circulans WL-12 revealed its evolutionary relationship to Serratia chitinase and to the type III homology units of fibronectin. J Biol Chem 265:15659-15665.
Zhong WF, Jiang LH, Yan WZ, Cai PZ, Zhang ZX and Pei Y (2003) Cloning and sequencing of chitinase gene from $\mathrm{Ba}$ cillus thuringiensis serovar israelensis. Acta Genetica Sinica 30:364-369.

Zhong WF, Wu J, Cai PZ and Yan WZ (2004) Cloning and sequencing of Cry1Aa13 gene from Bacillus thuringiensis subsp sotto. J Sichuan University (Natural Science Edition) 41:1050-1053.

Associate Editor: Darcy Fontoura de Almeida 